This item was submitted to Loughborough's Research Repository by the author.

Items in Figshare are protected by copyright, with all rights reserved, unless otherwise indicated.

\title{
A hypersonic plasma bullet train traveling in an atmospheric dielectric-barrier discharge jet
}

PLEASE CITE THE PUBLISHED VERSION

PUBLISHER

(C) American Institute of Physics

\section{VERSION}

VoR (Version of Record)

\section{LICENCE}

CC BY-NC-ND 4.0

\section{REPOSITORY RECORD}

Shi, J.J., Fangchun Zhong, Jing Zhang, D.W. Liu, and Michael G. Kong. 2019. "A Hypersonic Plasma Bullet Train Traveling in an Atmospheric Dielectric-barrier Discharge Jet”. figshare. https://hdl.handle.net/2134/5195. 
This item was submitted to Loughborough's Institutional Repository (https://dspace.lboro.ac.uk/) by the author and is made available under the following Creative Commons Licence conditions.

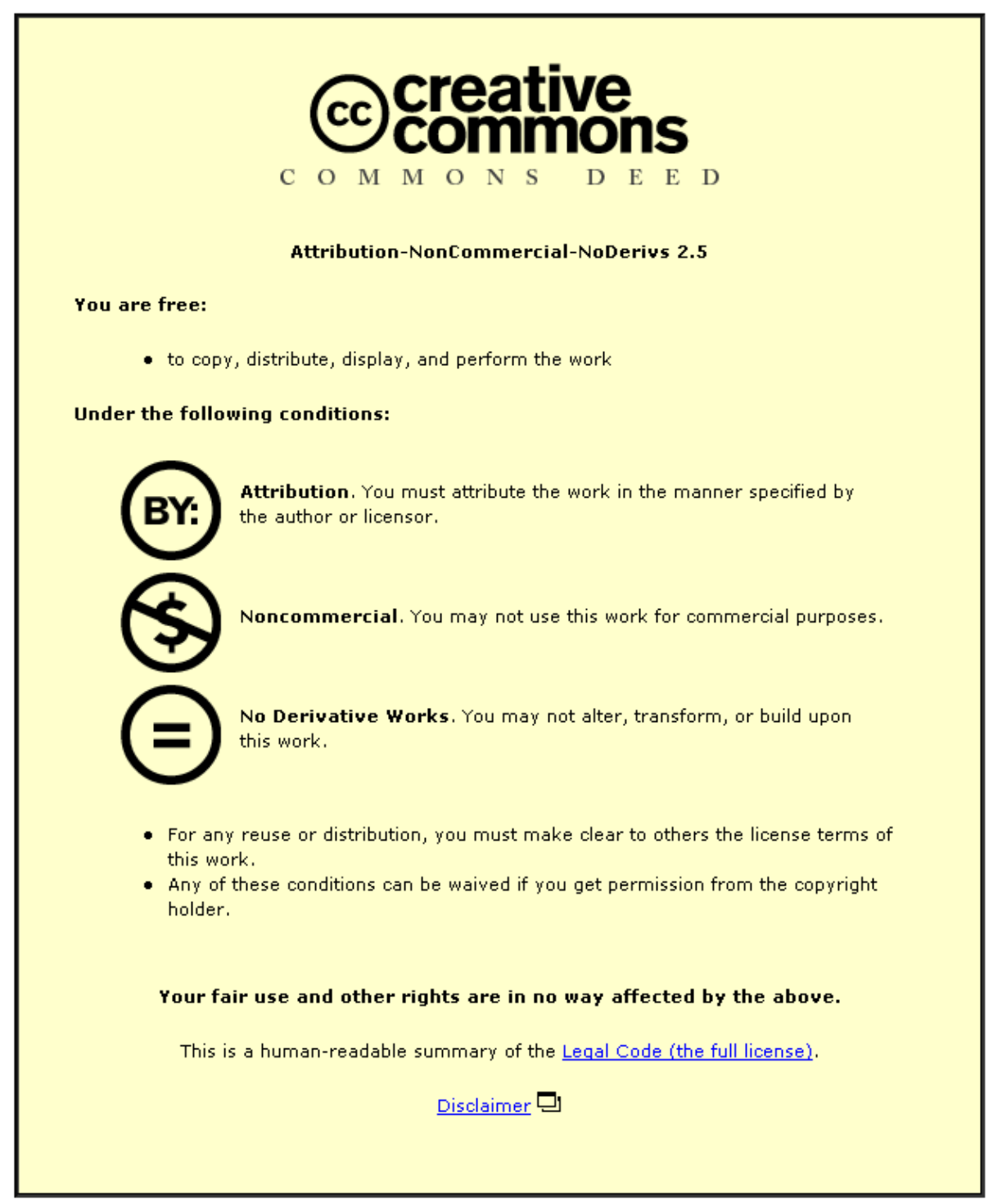

For the full text of this licence, please go to: http://creativecommons.org/licenses/by-nc-nd/2.5/ 


\title{
A hypersonic plasma bullet train traveling in an atmospheric dielectric-barrier discharge jet
}

\author{
Jianjun Shi, ${ }^{a)}$ Fangchun Zhong, and Jing Zhang \\ College of Science, Donghua University, Shanghai 201620, People's Republic of China \\ D. W. Liu and M. G. Kong \\ Department of Electronic and Electrical Engineering, Loughborough University, Loughborough, \\ Leicestershire LE11 3TU, United Kingdom
}

(Received 26 July 2007; accepted 3 December 2007; published online 15 January 2008)

\begin{abstract}
An experimental observation of fast-moving plasma bullets produced in an atmospheric dielectric-barrier discharge jet is reported in this paper. Nanosecond imaging suggests that the atmospheric discharge jet consists of a plasma bullet train traveling at a hypersonic speed from $7.0 \mathrm{~km} / \mathrm{s}$ to $43.1 \mathrm{~km} / \mathrm{s}$. Yet on a millisecond scale, the bullet train appears as a plasma jet of several centimeters long. The plasma bullets are produced through several possible mechanisms, the most likely of which is related to the ionization wave. Time and space resolved optical emission spectroscopy show that reactive plasma species can be delivered to different spatial sites with varying quantities. (C) 2008 American Institute of Physics. [DOI: 10.1063/1.2828551]
\end{abstract}

\section{INTRODUCTION}

Nonthermal atmospheric plasma jets have recently attracted growing interest, motivated by their considerable application potentials for material modification ${ }^{1-3}$ and biomedical applications. ${ }^{4,5}$ These plasma sources can be generated with sinusoidal excitation at $60 \mathrm{~Hz},{ }^{6} \mathrm{kHz}^{5,7-10} \mathrm{MHz},{ }^{1,4,11-14}$ and with repetitive nanosecond pulses at kilohertz. ${ }^{8,15}$ For many of their applications, nonthermal plasma jets offer a useful practicality of separating the plasma generation region from the application region thus facilitating access to an extensive range of reaction chemistry. ${ }^{7}$ However, possible mechanisms responsible for the production of atmospheric plasma jets remain largely unknown. Several recent reports suggest that atmospheric plasma jets could be formed through fast-moving plasma bullets. ${ }^{8-10,15}$ Further investigations are needed to progress to these preliminary studies and to advance the current understanding of atmospheric plasma jets. In this article, we present an experimental investigation of fast-moving plasma bullets formed in an atmospheric dielectric-barrier discharge jet.

\section{EXPERIMENTAL SETUP}

The atmospheric plasma jet used in this study was produced in a dielectric tube wrapped with a metal strip of $10 \mathrm{~mm}$ wide as the power electrode and a metal plate as the ground electrode placed a distance of $27 \mathrm{~mm}$ away from the nozzle of the dielectric tube, as shown in Fig. 1(a). The inner and outer diameters of the dielectric tube were 1.58 and $2.44 \mathrm{~mm}$, respectively. The electrodes were connected to a $20 \mathrm{kHz}$ sinusoidal voltage with an amplitude of $4 \mathrm{kV}$. A helium gas flow of 5 standard liters per minute (SLM) was fed through the dielectric tube and the resulting discharge was ejected out of the outlet of the dielectric tube, in the form of

\footnotetext{
${ }^{\text {a) }}$ Author to whom correspondence should be addressed. Electronic mail: JShi@dhu.edu.cn.
}

a plasma jet, into the surrounding ambient air. Therefore, it was essentially an atmospheric dielectric-barrier discharge jet, details of which have been reported elsewhere. ${ }^{5,7}$ Current and voltage were measured by a current probe (Tektronix P6021) and a voltage probe (Tektronix P6015A), and recorded on a digital oscilloscope (Tektronix TDS 3000B). To image the plasma jet on a nanosecond scale, an intensified charge coupled device (iCCD) camera (Andor i-Star DH720) was used with its triggering achieved using a pulse generator. Optical emission spectrum was measured with a spectrometer system (Andor Shamrock) with a focal length of $0.3 \mathrm{~m}$ and a grating of 600 grooves $/ \mathrm{mm}$.

\section{RESULTS AND DISCUSSIONS}

With the exposure time of the iCCD camera set at $1 \mathrm{~ms}$, the plume of the atmospheric dielectric-barrier discharge was imaged and it appeared as a plasma jet as shown in Fig. 1(a). Typical waveforms of discharge current and applied voltage are shown in Fig. 1(b). The applied voltage is predominately sinusoidal but with a small positive dc offset, and its positive and negative amplitudes are $3.94 \mathrm{kV}$ and $3.57 \mathrm{kV}$, respectively. The total current measured from the ground electrode is made of a displacement current and a discharge current, with the former being largely sinusoidal and the latter being essentially conductive current. The discharge current appears in Fig. 1(b) as a large spike superimposed onto a small sinusoidal displacement current. As the applied voltage has a positive dc offset, the current has a distinct peak of $1.28 \mathrm{~mA}$ in each positive half-cycle of the applied voltage, whereas its peak is barely visible in the negative half-cycles. Further increase of the applied voltage will lead to a significant current peak in both the positive and the negative half-cycles of the applied voltage, exhibiting the typical current-voltage characteristics of atmospheric dielectric-barrier discharges. ${ }^{16}$ The gate signal for triggering the iCCD camera is also shown in Fig. 1(b) and its time delay was used to correlate the bullet 


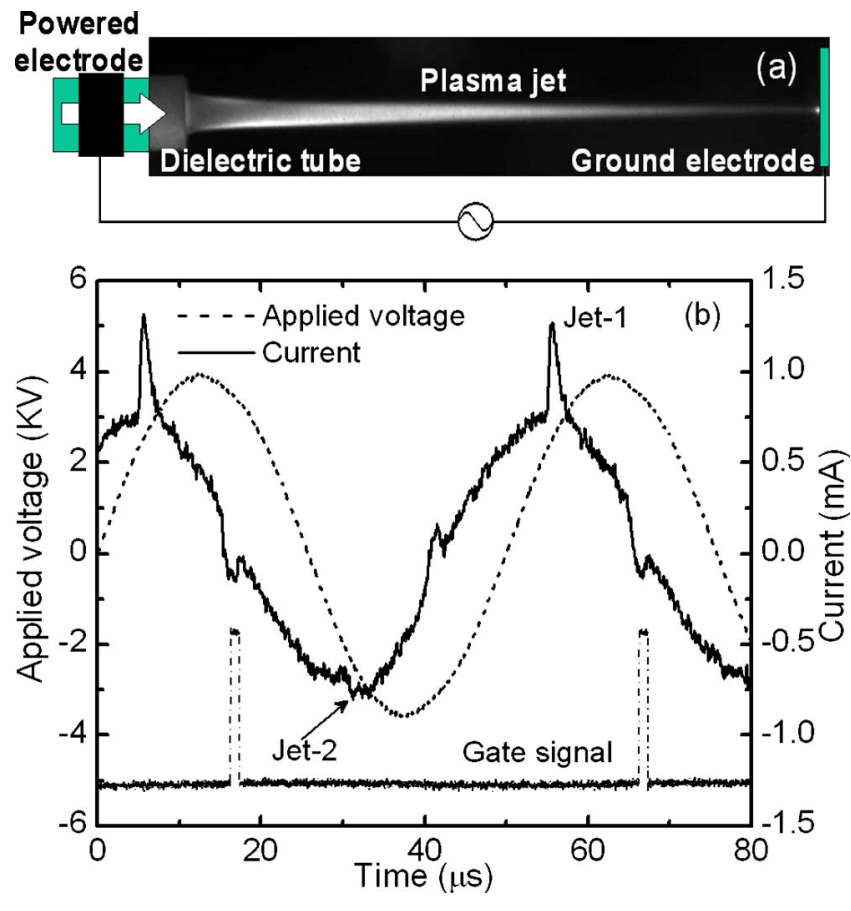

FIG. 1. (Color online) (a) Image of an atmospheric dielectric-barrier discharge jet with $1 \mathrm{~ms}$ exposure time and the schematic of its electrode unit; (b) current (solid) and voltage (dashed) waveforms of the plasma jet together with the iCCD triggering signal.

images directly to a specific time instant on the discharge current waveform. As indicated in Fig. 1(b), the plasma jet at the positive and negative half cycle of the applied voltage are referred to as Jet-1 and Jet-2, respectively. It is worth mentioning that the plasma plume can also be produced in the absence of the ground electrode.

Figure 2 shows a sequence of 20 images of the plasma jet, each with an exposure time of $1 \mathrm{~ns}$, during a positive half-cycle of the applied voltage (Jet-1) when the ground electrode was the instantaneous cathode. The time interval

\begin{tabular}{|c|c|c|c|}
\hline (a) & $38.6 \mu \mathrm{s}$ & $39.6 \mu \mathrm{s}$ & - (b) \\
\hline . & $38.7 \mu \mathrm{s}$ & $39.7 \mu \mathrm{s}$ & - \\
\hline$=$ & $38.8 \mu \mathrm{s}$ & $39.8 \mu \mathrm{s}$ & $=$ \\
\hline- & $38.9 \mu \mathrm{s}$ & $39.9 \mu \mathrm{s}$ & \\
\hline 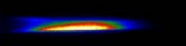 & $39.0 \mu \mathrm{s}$ & $40.0 \mu \mathrm{s}$ & \\
\hline- & $39.1 \mu \mathrm{s}$ & $40.1 \mu \mathrm{s}$ & \\
\hline & $39.2 \mu \mathrm{s}$ & $40.2 \mu \mathrm{s}$ & \\
\hline & $39.3 \mu \mathrm{s}$ & $40.3 \mu \mathrm{s}$ & \\
\hline & $-39.4 \mu \mathrm{s}$ & $40.4 \mu \mathrm{s}$ & \\
\hline & $39.5 \mu \mathrm{s}$ & $40.5 \mu \mathrm{s}$ & \\
\hline
\end{tabular}

FIG. 2. (Color online) Nanosecond images of Jet-1 with 1 ns exposure time of the plasma jet at different delay times from the rising edge of the iCCD gate signal.

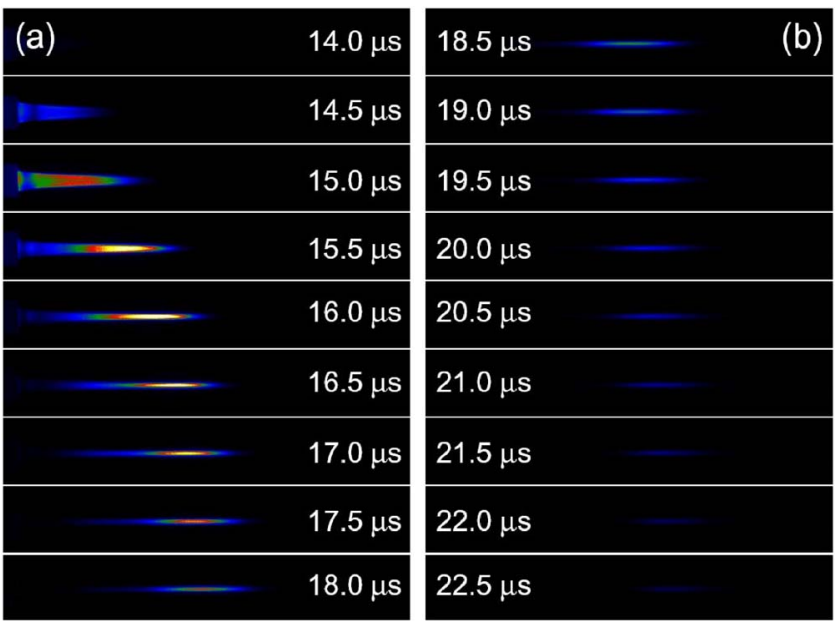

FIG. 3. (Color online) Nanosecond images of Jet-2 with 1 ns exposure time of the plasma jet at different delay times from the rising edge of the iCCD gate signal.

between any two successive images was fixed at $100 \mathrm{~ns}$. These nanosecond images suggest that the plasma jet shown in Fig. 1(a) with $1 \mathrm{~ms}$ exposure time was actually formed by a fast moving plasma bullet. In each pulse of the discharge current, one plasma bullet is produced and so over a sufficiently long period of time the atmospheric dielectric-barrier discharge jet is essentially a fast-moving train of plasma bullets with $1 /(2 \times 20) \mathrm{kHz}=25 \mu \mathrm{s}$ between two sequential plasma bullets. As a plasma bullet traveled from the outlet of the dielectric tube to the ground electrode, its optical signature changed its intensity and size. The time delay marked in Fig. 2 corresponds to the time difference between the gate signal and a point on the discharge current waveform in Fig. 1(b), and so at a time delay of $38.7 \mu \mathrm{s}$, when the discharge current start to go up, marks the emergence of a plasma bullet from the dielectric tube. At a time delay of $39.3 \mu \mathrm{s}$, the discharge current reached its peak value, and the intensity of the plasma bullet also became strongest. This suggests that during the rising phase of the discharge current, the plasma bullet grew and became stronger, as shown in Fig. 2(a). After that, the plasma bullet started to die off as the discharge current started to decline. During this current-falling phase, there were fewer and fewer sufficient energetic electrons to produce relevant excited plasma species resulting in a diminishing optical emission. Figure 2(b) shows the plasma bullet reaching the ground electrode and extinguished there. The time between the first and the last images in Fig. 2 is $2.0 \mu \mathrm{s}$, close to the current pulse width of $2.3 \mu \mathrm{s}$. Therefore the discharge current peak may be attributed to the moving of the plasma bullet across the discharge gap.

Although there is no large discharge current peak in the negative applied voltage phase, a weak plasma bullet (Jet-2) was observed during this voltage-falling phase. It is shown in Fig. 3 that a sequence of 18 images of Jet-2. As Jet-2 was much weaker and traveling at a slower speed than Jet-1, which will be shown later, the time interval between any two successive images was fixed at $500 \mathrm{~ns}$ and the image intensity was enhanced. Compared to the plasma bullet in Jet-1, the plasma bullet in Jet-2 was longer and started to die off in 


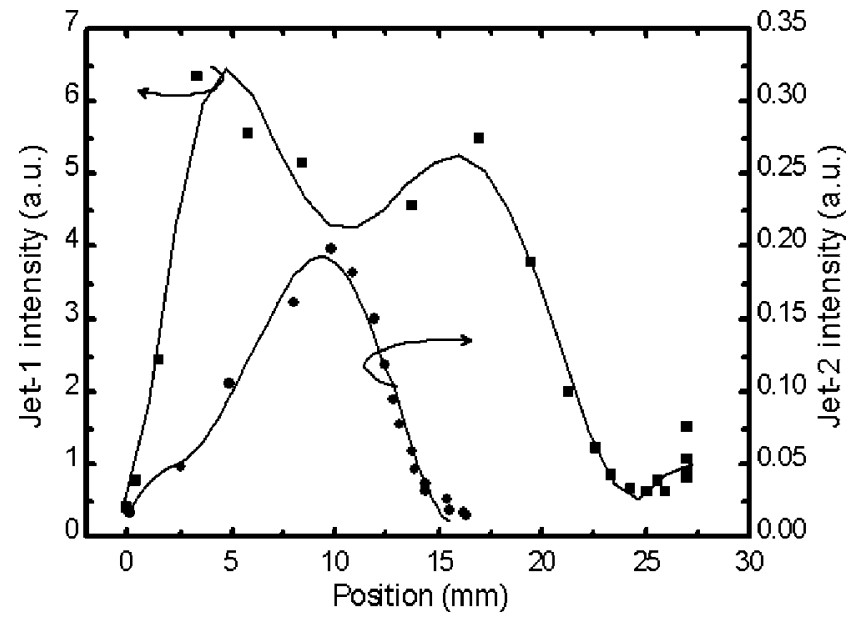

FIG. 4. The maximum optical intensity of the plasma bullet in Jet-1 and Jet-2 as a function of the distance from the exit of the dielectric tube.

the middle of the discharge gap well before reaching the ground electrode. It is worth mentioning that the lifetime of Jet-2 $(\sim 9 \mu \mathrm{s})$ is much longer than that of Jet-1 $(2.0 \mu \mathrm{s})$.

Figure 4 shows the position dependence of the optical emission intensity of the plasma bullet, indicating clearly that it is much brighter in the middle than the two ends. For Jet-1, the position dependence has an interesting profile of double humps with the two maximum optical intensities occurring at $2.5 \mathrm{~mm}$ and $17.5 \mathrm{~mm}$, respectively. The reason for this interesting double hump spatial profile remains unknown; further measurements with better spatiotemporal resolution will be necessary in future studies. This doublehumped profile contrasts with the position dependence of the bullet size that has the largest bullet centered around $10 \mathrm{~mm}$. The intensity of the plasma bullet reaches its maximum of 6.3 (arbitrary unit). In Jet-2, the plasma bullet traveled a longer distance of $9.8 \mathrm{~mm}$ before reaching its maximum intensity of 0.20 and was extinguished at $16.3 \mathrm{~mm}$ from the nozzle of the dielectric tube. This clearly shows that the optical emission intensity of Jet-2 is much weaker than that of Jet- 1 with the ratio between their maximum value being $6.3 / 0.2=31.5$. These results suggest that the plasma jet was dominated by Jet-1 although it was sustained over a longer period in Jet-2.

Figure 5 shows the position dependence of the bullet velocity, estimated from the sequence of nanosecond bullet images. In Jet-1, it is found that the bullet speed started at about $7.5 \mathrm{~km} / \mathrm{s}$ at the exit of the dielectric tube, reached its peak of $43.1 \mathrm{~km} / \mathrm{s}$ at $13.8 \mathrm{~mm}$, and then reduced to $7.0 \mathrm{~km} / \mathrm{s}$ near the ground electrode. As the plasma bullet in Jet-2 increased in size, the bullet speed became difficult to estimate. At a position of $4.9 \mathrm{~mm}$, the bullet reached the maximum speed of $0.5 \mathrm{~km} / \mathrm{s}$, which is about two orders of magnitude lower than the maximum bullet speed in Jet-1. After that, the bullet speed decreased to $0.08 \mathrm{~km} / \mathrm{s}$ at $16.1 \mathrm{~mm}$ from the nozzle of the dielectric tube. In other words, both plasma bullets accelerate first and then decelerate towards the ground electrode. The maximum bullet velocity is reached at approximately the same position as that where the largest bullet size is reached.

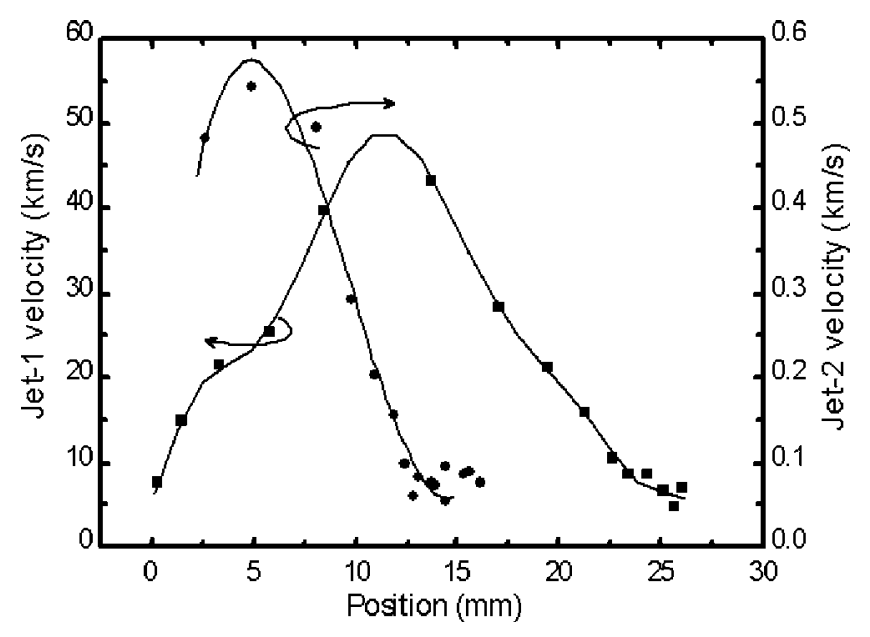

FIG. 5. The velocity of the plasma bullet in Jet-1 and Jet-2 as a function of the distance from the exit of the dielectric tube.

As the plasma jet is dominated by Jet-1, the characteristics of Jet-1 are explained in the following. The speed of the plasma bullet in Jet-1 is at the very high end of the hypersonic range. The gas flow rate of helium in the dielectric tube is estimated to be $10.6 \mathrm{~m} / \mathrm{s}$, some three orders of magnitude lower than the bullet speed. Further experiments (not shown here) suggest that the speed of the plasma bullet is independent of flow rate of fed helium gas. Therefore, it is unlikely that the plasma bullets are propelled by the gas flow. By assuming that the electric field was uniform and set up by the applied voltage, the drift velocity of helium atom ions and molecular ions are estimated to be $133.4 \mathrm{~m} / \mathrm{s}$ and $319.6 \mathrm{~m} / \mathrm{s}$ thus suggesting their minor roles, if any, in the production of the plasma bullets. Again assuming a uniform electric field, the electron drift velocity is found to be $15.0 \mathrm{~km} / \mathrm{s}$, similar to the bullet velocity of $7.0-48.7 \mathrm{~km} / \mathrm{s}$. However, it does not explain why plasma bullets, if composed of negatively charged electrons, move towards the instantaneous cathode.

It is possible that the plasma bullet is produced by the ionization wave front. ${ }^{17}$ There are three mechanisms through which to contribute to the ionization front velocity, namely the electron diffusion, the ponderomotive force and the breakdown wave. The ionization wave front velocity due to electron diffusion can be expressed as $v=2\left[v_{i} D_{a}\right]^{1 / 2}$, ${ }^{17}$ where $v_{i}$ is the ionization frequency and $D_{a}=10^{4} / p$ is the ambipolar diffusion coefficient with $p$ being the gas pressure in Torr. As the cross-sectional area of the plasma jet is about $0.02 \mathrm{~cm}^{2}$, the maximum current density is $64.0 \mathrm{~mA} / \mathrm{cm}^{2}$ at the peak current of $1.28 \mathrm{~mA}$. Given that the discharge event lasts for $2.3 \mu$ s during which the applied voltage changes very little, this transient discharge may be approximated as a dc discharge occurring at the discharge current peak. According to a simple model for dc atmospheric pressure glow discharges in helium, ${ }^{18}$ the maximum electron energy is $32.2 \mathrm{eV}$ at a current density of $64.0 \mathrm{~mA} / \mathrm{cm}^{2}$. In turn, this leads to an estimate of the frequency of direct helium ionization by electrons at $1.45 \times 10^{11} \mathrm{~s}^{-1}$. ${ }^{19}$ With these parameters, the velocity of the ionization wave is estimated to be $27.7 \mathrm{~km} / \mathrm{s}$. This is close to the maximum plasma bullet velocity of $43.1 \mathrm{~km} / \mathrm{s}$ in Fig. 5. Given that only the electron diffusion mechanism is 

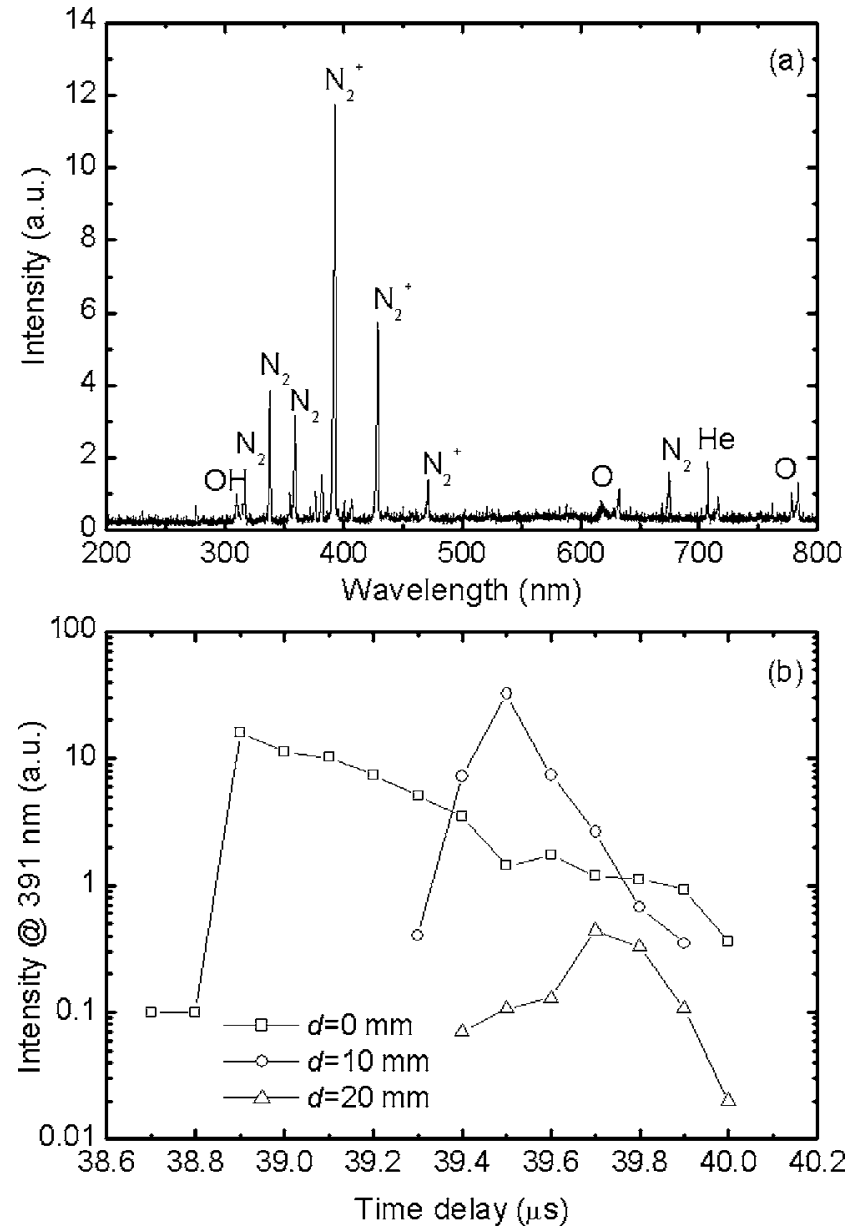

FIG. 6. (a) The optical emission spectrum from the plasma jet taken at $10 \mathrm{~mm}$ away from the exit of the dielectric tube and with $1 \mathrm{~ms}$ exposure time; (b) the time-resolved $391 \mathrm{~nm}$ line measured with $5 \mathrm{~ns}$ exposure time and at 0,10 , and $20 \mathrm{~mm}$ away from the exit of the dielectric tube.

considered and our estimate of the electron energy is likely to be simplistic, this agreement is particularly encouraging. The two other mechanisms of the ionization wave, namely the ponderomotive force and the breakdown wave, depend on the gradient of the electric field. Although the current lack of established experimental techniques for measuring the electric field has prevented a reliable estimate of their contributions to the ionization wave in this study, it is conceivable that they are likely to add to the ionization front velocity of $27.7 \mathrm{~km} / \mathrm{s}$ estimated from electron diffusion only and thus bring the overall speed of the ionization wave front closer to the maximum plasma bullet speed of $43.1 \mathrm{~km} / \mathrm{s}$. In short, it is likely that plasma bullets are produced through the ionization wave front. An atmospheric pressure plasma plume generated by submicrosecond voltage pulses was studied by others. These authors proposed a model based on photoionization to explain the dynamics of the plasma plume. ${ }^{15}$ It suggests that more must be done in order to understand the relative contributions of different mechanisms being put forward.

To characterize the plasma bullets further, their optical emission spectrum is shown in Fig. 6(a) with an exposure time of $1 \mathrm{~ms}$ and the optical probe placed at $10 \mathrm{~mm}$ from the exit of the dielectric tube. It shows that the dominated emis- sion lines are from the nitrogen excited species. Corresponding to the $R$ branch of the first negative system of $\mathrm{N}_{2}^{+}(0-0)$, the $391 \mathrm{~nm}$ line is the strongest in Fig. 4(a). The timeresolved $391 \mathrm{~nm}$ line was measured with an exposure time of $5 \mathrm{~ns}$, and its spatial resolution was obtained by repeating the time-resolved measurement at 0,10 , and $20 \mathrm{~mm}$ away from the exit of the dielectric tube. The peak optical intensities of the $391 \mathrm{~nm}$ line are seen in Fig. 6(b) to occur at a delay time of $38.9 \mu \mathrm{s}, 39.5 \mu \mathrm{s}$, and $39.7 \mu \mathrm{s}$, respectively, when the plasma bullet travels across the three observation positions. As the $391 \mathrm{~nm}$ line is related to direct ionization of the molecular nitrogen with a threshold electron energy of $18.7 \mathrm{eV},{ }^{19,20}$ its temporal profiles at these three positions suggest the presence of energetic electrons in the electrode gap. With plenty in the $0-10 \mathrm{~mm}$ region, these energetic electrons are most numerous at $d=10 \mathrm{~mm}$. Beyond $d$ $=10 \mathrm{~mm}$, the number of energetic electrons starts to decrease rapidly. This trend is consistent with that of the bullet images in Fig. 2 and their characterization in Figs. 4 and 5. It is also consistent with the suggestion that electron diffusion and hence the ionization wave are likely to be responsible for hypersonic plasma bullets. The spatial variation of the 391 line in Fig. 4(b) and more generally reactive plasma species represents a useful application flexibility of delivering reactive plasma species of varying quantities to different processing locations.

\section{SUMMARY}

In summary, the atmospheric dielectric barrier discharge jet has been shown to be formed by a hypersonic plasma bullet train. It has been established that their production is likely to be through the ionization wave and that they can be used to deliver reactive plasma species to different spatial locations.

\section{ACKNOWLEDGMENTS}

The authors are grateful for the financial support from the Engineering and Physical Sciences Research Council, UK and the National Science Foundation of China (Grant No. 10745004).

${ }^{1}$ J. Y. Jeong, S. E. Babayan, V. J. Tu, J. Park, I. Henins, R. F. Hicks, and G. S. Selwyn, Plasma Sources Sci. Technol. 7, 282 (1998).

${ }^{2}$ M. Benmansour, M. Nikravech, D. Morvan, J. Amouroux, and J. Chapelle, J. Phys. D 37, 2966 (2004).

${ }^{3}$ Q. Chen, Y. Zhang, E. Han, and Y. Ge, Plasma Sources Sci. Technol. 14, 670 (2005)

${ }^{4}$ E. Stoffels, I. E. Kieft, and R. E. J. Sladek, J. Phys. D 36, 2908 (2003).

${ }^{5}$ X. T. Deng, J. J. Shi, G. Shama, and M. G. Kong, Appl. Phys. Lett. 87, 153901 (2005)

${ }^{6}$ M. H. Guerra-Mutis, C. V. Pelaez U., and R. H. Cabanzo, Plasma Sources Sci. Technol. 12, 165 (2003).

${ }^{7}$ J. L. Walsh, J. J. Shi, and M. G. Kong, Appl. Phys. Lett. 88, 171501 (2006).

${ }^{8}$ M. Laroussi and X. Lu, Appl. Phys. Lett. 87, 113902 (2005).

${ }^{9}$ J. Kedzierski, J. Engemann, M. Teschke, and D. Korzec, Solid State Phenom. 107, 119 (2005)

${ }^{10}$ M. Teschke, J. Kedzierski, E. G. Finantu-Dinu, D. Korzec, and J. Engemann, IEEE Trans. Plasma Sci. 33, 310 (2005).

${ }^{11}$ S. Wang, V. S. Gathern, and H. F. Dobele, Appl. Phys. Lett. 83, 3272 (2003). 
${ }^{12}$ J. Janca, M. Klima, P. Slavicek, and L. Zajickova, Surf. Coat. Technol. 116, 547 (1999).

${ }^{13}$ M. Chichina, Z. Hubicka, O. Churpita, and M. Tichy, Plasma Processes Polym. 2, 501 (2005).

${ }^{14}$ T. Ichiki, T. Koidesawa, and Y. Horiike, Plasma Sources Sci. Technol. 12, S16 (2003)

${ }^{15}$ X. P. Lu and M. Laroussi, J. Appl. Phys. 100, 063302 (2006).
${ }^{16}$ M. G. Kong and X. T. Deng, IEEE Trans. Plasma Sci. 31, 7 (2003).

${ }^{17}$ A. Bohle, O. Ivanov, A. Kolisko, U. Kortshagen, H. Schluter, and A. Vikharev, J. Phys. D 29, 369 (1998).

${ }^{18}$ J. J. Shi and M. G. Kong, J. Appl. Phys. 94, 5504 (2003).

${ }^{19}$ X. Yuan and L. L. Raja, IEEE Trans. Plasma Sci. 31, 495 (2003).

${ }^{20}$ K. V. Kozlov, H. E. Wagner, R. Brandenburg, and P. Michael, J. Phys. D 34, 3164 (2001). 